\title{
Study on Assembly Precision Reliability Assessment for Industrial Sensing Devices
}

\author{
Tian Fan ${ }^{1, a}$, Wei Tao ${ }^{1}$, Hanxian $\mathrm{Hu}^{1}$ and Wei Liu ${ }^{1}$ \\ CRRC QINGDAO SIFANG CO., LTD., Shandong Qingdao 266111, China
}

\begin{abstract}
This paper investigates the method of assembly precision reliability assessment for industrial sensing devices. The part variation is formulated by small displacement torsor. The assembly error model is developed through the approach of pose transformation matrix, then the error transfer between the parts is analyzed and the assembly error is calculated. The concept of assembly precision reliability is proposed. Monte Carlo simulation is adopted to calculate the reliability, after that the procedure of assembly precision reliability is determined. Case study of a laser sensor is provided therefore the effectiveness of the proposed models is verified.
\end{abstract}

\section{Introduction}

Assembly precision is a crucial technique target for industrial sensing devices. As the devices play important role in industrial network system, and different devices can be used to measure diverse physical quantities, such as length, distance, vibration, speed. Meanwhile, assembly precision of industrial sensing devices has an important impact on the measuring precision. Therefore, the study on assembly precision reliability assessment for industrial sensing devices has potential value of engineering application.

Manufacturing error and assembly error are gradually introduced and accumulated in assembly process, which eventually affect the assembly precision reliability of the devices. Therefore, the study of assembly precision reliability assessment should be based on the propagation and accumulation analysis of error sources.

Some methods of assembly error transfer commonly used include Stream of Variation (SoV)1, state space method1 2, multi-body system theory4, etc. In the early, Mantripragada5 presented a state transition model of assembly and concepts from control theory to model variation propagation and control during assembly. Also, a systematic study regarding assembly variation modeling in automobile assemblies is conducted at the University of Michigan. HU1 developed a methodology to evaluate the dimensional variation propagation in a multi-station compliant assembly system based on linear mechanics and a state space representation. Literature 25 developed process state space model based on SoV. Liu7 adopted SoV and Statistical Process Control (SPC) methodologies respectively to analyze the variation reduction for multistage manufacturing processes. Yu8 established a geometric error propagation model by the theory of multi-body system and homogeneous transformation method (HTM). Tan, et al8 investigated surface variation of compliant Parts, and developed a comprehensive approach of geometrical covariance modeling. The industrial robot flexible assembly technology for the plane drilling and riveting was presented in literature9. Tang 11 introduced a new geometric error modeling approach for multi axes system (MAS) based on SoV, especially for multi-axis precision stage. Cao12 investigated a vibration propagation model based on key point.

Many researches have been done in the field of mechanical precision reliability. Literature8 took reliability as the index of machining precision, and proposed the method of precision assignment based on reliability analysis. Besides, response surface method was adopted to calculate precision reliability in this paper. Based on the theory of mechanical precision design and mechanical reliability design, Song established the model of machine tool transmission precision reliability, and studied the main factors affecting the transmission precision of $\mathrm{CNC}$ machine tools 13

Reliability theory has been widely applied in the field of mechanical structure and precision, while there is still no general assembly precision reliability evaluation system that can be used for industrial sensing devices. In this paper, combined with the actual need of Laser sensor assembly, the product assembly error transfer model is established and reliability assembly precision is evaluated. The model proposed considers part dimension, product structure, assembly error and other factors systematically. The study in this paper can provide an effective theoretical method for the assembly precision reliability for industrial sensing devices.

\section{Assembly Error Transfer Model}

a Corresponding author: 1064272117@qq.com 
Assembly is the process of connecting parts together by mechanical connection mode. During assembly, error is transferred and accumulated through joint surface. In this section, part error is presented by unified expression and assembly error transfer model is established based on the HTM.

\subsection{Part variation}

In this paper, small displacement torsor(SDT) is presented to describe the part variation as this representation can be conveniently transformed into a transfer matrix form, then the relationship between the error and the target precision is obtained through the transformation of HTM.

Parts variation includes direction error along $x, y$ and $z$ axes and orientation error about the $x, y$ and $z$ axes, which can be expressed as error vector. For a part $G$, error vector consists of small linear shift along the axes of a point $g$ on the part $G$ and small angular shift about the axes of the whole part:

$$
\left\{\begin{aligned}
\mathbf{G}(g) & =\left[\begin{array}{lll}
\Delta X_{g}(G) & \Delta Y_{g}(G) & \Delta Z_{g}(G)
\end{array}\right]^{\mathrm{T}} \\
\mathbf{G}^{\prime} & =\left[\begin{array}{lll}
\Delta \alpha(G) & \Delta \beta(G) & \Delta \theta(G)
\end{array}\right]^{\mathrm{T}}
\end{aligned}\right.
$$

Where $\mathbf{G}(g)$ represents position error vector of the part; G'represents the direction error vector of the part; $\Delta X_{g}(G), \Delta Y_{g}(G), \Delta Z_{g}(G)$ represent small translational displacement along the axes of the point $g$ on part $G$, respectively; $\Delta \alpha(G), \Delta \beta(G), \Delta \theta(G)$ represent small rotational displacement about the axes of part $G$.

\subsection{Assembly Error Transfer Model}

\subsubsection{Transformation matrix}

Position and orientation transformation of parts under different coordinate system can be expressed by HTM:

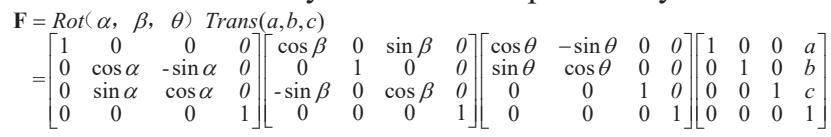

Where Trans is translation transformation; $a, b, c$ is translational displacement along the $x, y, z$ axes during coordinate transformation respectively; Rot is rotation transformation; $\alpha, \beta, \theta$ is rotational displacement about the $x, y, z$ axes during coordinate transformation respectively.

When assembly error exists, there will be small translational displacement along the $x, y, z$ axes and small rotational displacement about the $x, y, z$ axes during coordinate transformation; Ignoring second and third order components in formula (2), the error transformation matrix can be obtained

$$
\Delta \mathbf{F}=\left[\begin{array}{cccc}
1 & -\sin \Delta \gamma & \sin \Delta \beta & \Delta a \\
\sin \Delta \gamma & 1 & -\sin \Delta \alpha & \Delta b \\
-\sin \Delta \beta & \sin \Delta \alpha & 1 & \Delta c \\
0 & 0 & 0 & 1
\end{array}\right]=\left[\begin{array}{cccc}
1 & -\Delta \gamma & \Delta \beta & \Delta a \\
\Delta \gamma & 1 & -\Delta \alpha & \Delta b \\
-\Delta \beta & \Delta \alpha & 1 & \Delta c \\
0 & 0 & 0 & 1
\end{array}\right]
$$

And transformation matrix can be written as

$$
\mathbf{F}^{\prime}=\Delta \mathbf{F} \cdot \mathbf{F}
$$

\subsubsection{Construction of Assembly Error Transfer Coordinate System}

The assembly error propagation can be described as error transfer between joint surfaces in the assembly process. Joint surface refers to a surface on a part which has joint relationship with a surface on another part during the assembly process. Joint surface has a variety of types, such as plane, cylinder, cone, sphere, etc. Among these, planar surface (shown as the join surface between part 1 and part 2 in Fig.1) and cylindrical surface (shown as the join surface between part 2 and part 3 in Fig.1) are the most common features[14].

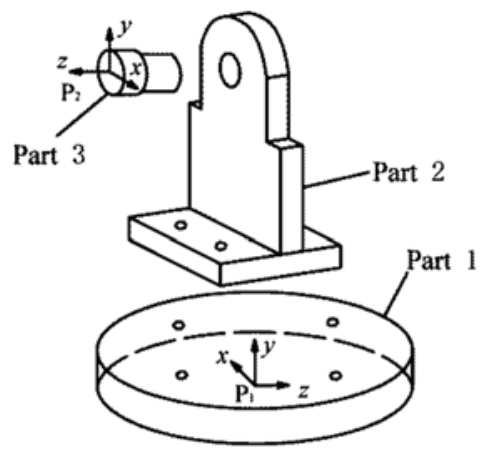

Figure 1. Schematic diagram of parts assembly

\subsubsection{Calculation of Assembly Error Transfer}

During the assembly error transfer model building, the first step is to establish the error transfer coordinate system. As for a machine, if there are $t$ parts to be assembled, we need to establish $t+1$ coordinate systems, including the global reference coordinate system defined as $\mathrm{P}_{0}$, the target coordinate system defined as $\mathrm{P}_{t}$, the error transfer coordinate between parts defined as $\mathrm{P}_{1}, \mathrm{P}_{2}, \ldots \mathrm{P}_{t-1}$.

As for a middle part $I$, its position and orientation transformation relationship between coordinate system $\mathrm{P}_{i}$ and $\mathrm{P}_{i-1}$ can be represented as:

$$
\left\{\begin{array}{l}
\mathbf{U}_{i-1}^{i_{m}}=\mathbf{V}_{i} \mathbf{U}_{i_{m}} \\
\mathbf{U}_{i-1}^{i_{m}}=\left(\Delta \mathbf{M}_{i}+\Delta \mathbf{N}_{i}\right) \mathbf{V}_{i} \mathbf{U}_{i_{m}}
\end{array}\right.
$$

Where $\mathbf{U}_{i_{m}}$ is the ideal coordinate vector of point $m$ on part $i$ under $\mathrm{P}_{i} ; \mathbf{V}_{i}$ is position and orientation transformation matrix of point $j$ between the two adjacent coordinate systems; $\mathbf{U}_{i-1}^{i_{m}}$ is the ideal coordinate vector of point $m$ on part $i$ under $\mathrm{P}_{i-1} ; \Delta \mathbf{M}_{i}$ is position and orientation transformation matrix of manufacturing error; $\Delta \mathbf{N}_{i}$ is position and orientation transformation matrix of assembly error; $\mathbf{U}_{i-1}^{i_{m}}$ is the actual coordinate vector of point $m$ on part $i$ under $\mathrm{P}_{i-1}$. 
According to Eq. (5), combined with a series of position and orientation transformation matrix, the normal coordinate vector and the actual coordinate vector of point $I$ on the target part $t$ under $\mathrm{P}_{t}, \mathrm{P}_{t-1}, V_{N-2}, P_{t-2} \ldots P_{\mathrm{o}}$ can be obtained:

$\left\{\mathbf{U}_{o}^{t_{t}}=\mathbf{V}_{1}, \mathbf{V}_{2} \cdots \mathbf{V}_{t-1} \mathbf{V}_{t} \mathbf{U}_{t_{t}}\right.$

$\left\{\mathbf{U}_{o}^{o}=\left(\Delta \mathbf{M}_{1}+\Delta \mathbf{N}_{1}\right) \mathbf{V}_{1}\left(\Delta \mathbf{M}_{2}+\Delta \mathbf{N}_{2}\right) \mathbf{V}_{2} \cdots\left(\Delta \mathbf{M}_{t-1}+\Delta \mathbf{N}_{t-1}\right) \mathbf{V}_{t-1}\left(\Delta \mathbf{M}_{t}\right) \mathbf{V}_{t} \mathbf{U}_{t_{j}}\right.$

The difference between the actual coordinate value and the ideal coordinate value of the point $I$ in the global coordinate system is the error vector of the target part, denoted by $\Delta \mathbf{U}_{o}^{t_{I}}$.

$$
\Delta \mathbf{U}_{o}^{t_{I}}=\mathbf{U}_{o}^{t_{I}}-\mathbf{U}_{o}^{t_{I}}
$$

\section{Analysis of assembly precision reliability}

\subsection{Definition of Assembly Precision Reliability}

Assembly precision reliability refers to the probability that products meet the design requirements under regular assembly condition. Assumes that the calculation of the actual assembly precision is $\Delta \mathrm{S}$, the range of the target assembly precision is between $\Delta S_{\text {- }}$ and $\Delta S_{+}$. If $\Delta S_{-} \leq \Delta S \leq \Delta S_{+}$, the actual precision meets the known requirement. Otherwise doesn't meet the requirement. Set the sample size $L$ (to ensure the sampling accuracy, $L$ should be large enough), $l$ is the number that sample meets the requirement. The assembly precision reliability can be obtained as:

$$
R=\frac{l}{L} \cdot 100 \%
$$

\subsection{Calculation of Assembly Precision Reliability Based on Monte Carlo Simulation Method}

On the basis of the definition of assembly precision reliability, Monte Carlo simulation method is adopted to calculate the precision reliability data in this paper.

The error sources that affect the assembly precision are considered as random variables, then the probability distribution and parameters of the known error sources are obtained. Therefore, the assembly precision reliability can be solved by Monte Carlo simulation. The solution steps are as follows:

Step 1: Set the calculation object and specified precision reliability according to the actual requirement.

Step 2: Establish the assembly error transfer model of industrial sensing devices based on the assembly structure of the object. Therefore the actual value of assembly error can be calculated.

Step 3: Determine whether the actual error of the analysis object is within the specified limits. If the actual error is within the prescribed range, the effective simulation number needs to be increased once; otherwise, the number keeps unchanged. When the judgment is completed, return to Step 2 to continue the next round of calculations until the number of simulation reaches $L$.

Step 4: Output the result of assembly precision reliability.

The assembly precision reliability is an important indicator to evaluate the assembly quality of devices, and its calculation results can be used as a quantitative index to assess the assembly precision of industrial sensing devices.

\section{Case Study}

In this section, the proposed methodology is illustrated with an example of a laser sensor, the parts and basic dimensions of which is shown in Fig.2. Assumes that all parts of the sensor are at the given location in non-operating mode, dimensional tolerance of which is $\pm 0.05 \mathrm{~mm}$, geometric tolerance is $0.01 \mathrm{~mm}$. The target of the assembly precision is that the reliability of direction error of working part in each direction has to be not less than $95 \%$.

Lower surface on the base of the base is treated as global benchmark surface. The origin of each coordinate system is shown in Table 1, and each coordinate system is parallel to the global coordinate system $\mathrm{P} 0$.

The data in Table 2 shows that the mean deviation of the direction tends to accumulate during assembly.

The direction error reliability in $x, y$ and $z$ directions is $96.52 \%, 95.36 \%$ and $94.37 \%$, respectively, calculated by MATLAB simulation. The results show the direction error of $x$ and $y$ is in accord with the requirement of assembly precision, while the precision of $z$ direction is not satisfied with the requirement. According to the state variables calculated, the error state of the drilling machine can be estimated and predicted.

The difficulty of achieving different precision is different. When setting the target precision value of assembly, it is necessary to weigh the difficulty, cost and function realization, and so on. As for the assembly precision not conforming to the requirements, taking higher manufacture precision and other measures can reduce the assembly error of the parts. 

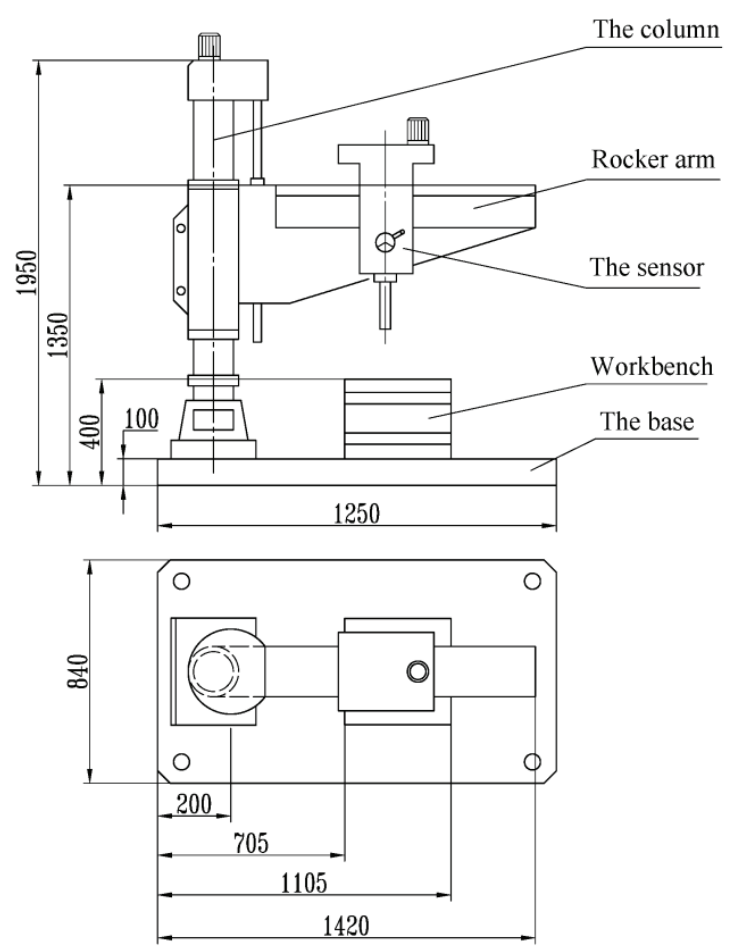

Fig.2 A frame model of a laser sensor

Table.1 The origin of coordinate system with respect to the global coordinate system $/ \mathrm{mm}$

\begin{tabular}{cccc}
\hline & $x$ & $y$ & $z$ \\
\hline $\mathrm{P}_{0}$ & 0 & 0 & 0 \\
$\mathrm{P}_{1}$ & 420 & 150 & 100 \\
$\mathrm{P}_{2}$ & 420 & 150 & 1350 \\
$\mathrm{P}_{3}$ & 420 & 1262.5 & 1350 \\
$\mathrm{P}_{4}$ & 420 & 1262.5 & 650 \\
\hline
\end{tabular}

Table.2 Mean of direction error of the parts during assembly

\begin{tabular}{ccccc}
\hline & The base & The column & Rocker arm & The sensor \\
\hline$\alpha / \mathrm{rad}$ & -0.00328 & 0.00175 & 0.00236 & 0.00278 \\
$\beta / \mathrm{rad}$ & 0.00192 & 0.00143 & 0.00201 & 0.00257 \\
$\theta / \mathrm{rad}$ & 0.00273 & 0.00230 & 0.00279 & 0.00280 \\
\hline
\end{tabular}

\section{Conclusion}

In this paper, the assembly error model is established by the position and orientation transformation matrix. Besides, the definition and the numerical calculation method of assembly precision reliability are given, which provides a theoretical basis for the reliability analysis of assembly precision. Through the assembly process analysis of a laser sensor, the validity of the method above is verified.

In the future study, we can further develop the sensitivity analysis of various error sources, and realize the optimization and improvement of the assembly precision by the reasonable allocation of the tolerances.

\section{References}

1. $\mathrm{S} \mathrm{Hu}, \mathrm{Y}$ Koren. CIRP ANN-MANUF TECHN, 46 (1997) : 1-6

2. J Jin, J Shi. J MANUF SCI E-T ASME, 121(1999):756-762

3. Y Ding, D Ceglarek, J Shi. JUSFA, (2000): 23-26

4. J Chen. INT J MACH TOOL MANU, 35(1995): 593-605

5. R Mantripragada, D Whitney. IEEE T ROBOTIC AUTOM, 15(1999): 124-140

6. S Zhou, Q Huang, J Shi. EEE T ROBOTIC AUTOM, 19(2003): 296-309

7. J Liu. QUAL RELIAB ENG INT, 26(2010): 645-661

8. Z Yu, Z Liu, Y Ai, et al. CHIN J MECH ENG, 49(2013): 142-151

9. C Tan, D Hou. Transactions of University of Aeronautics and Astronautics, 3(2014)

10. J Shen, W Tian. J NANJING U AERONAUT ASTRONAUTICS, 46(2014): 181-189

11. H Tang, J Duan, $\mathrm{S}$ Lan, et al. INT J MACH TOOL MANU, 92(2015):41-51

12. Y Cao, B Li, J Guan, et al. P I MECH ENG B-J ENG, 229(2015): 1164-1179

13. J Song, H Liu, D Yuan, et al. CHIN MECH ENG, 7(2011): 785-789

14. Yi G, et al. J. Zhejiang Univ, 40(2006): 921-926 